\title{
Knowledge transformers: A link between learning and creativity
}

\author{
SIANG KOK SIM ${ }^{1}$ AND ALEX H.B. DUFFY ${ }^{2}$ \\ ${ }^{1}$ School of Mechanical and Production Engineering, Nanyang Technological University, Singapore 639798, Republic of Singapore \\ ${ }^{2}$ CAD Centre, University of Strathclyde, Glasgow G1 1XJ, United Kingdom
}

(RECEIVED October 1, 2003; AcCePted July 7, 2004)

\begin{abstract}
The purpose of this paper is to investigate whether knowledge transformers that are featured in the learning process are also present in the creative process. First, this was achieved by reviewing accounts of inventions and discoveries with the view of explaining them in terms of knowledge transformers. Second, this was achieved by reviewing models and theories of creativity and identifying the existence of the knowledge transformers. The investigation shows that there is some evidence to show that the creative process can be explained through knowledge transformers. Hence, it is suggested that one of links between learning and creativity is through the knowledge transformers.
\end{abstract}

Keywords: Creativity; Discovery; Invention; Knowledge Transformers; Learning

\section{INTRODUCTION}

Creativity is considered the ultimate of human qualities, one of the key measures of intelligence that separates us from the rest of the animal kingdom (Goldenburg \& Mazursky, 2002). Our ability to create, to innovate, is believed to be godlike, described by some religions as one of the divine qualities endowed to man, who was created in the image of God, the Creator. The question that has perplexed many researchers and scholars in the study of creativity is what is creativity? It has been suggested that there are more than 200 definitions in literature alone (Weisberg, 1993; DasGupta, 1994). Understanding what is creativity has been the focus of many research and academic studies across many disciplines, from psychology to art to medicine. Taylor (1988), in analyzing more than 50 definitions, categorized the various interpretations into one of several classes (Dasgupta, 1994):

1. Gestalt type: the major emphasis is on the recombination of ideas or the restructuring of a Gestalt.

2. End product oriented: according to which creativity is a process that results in a novel output or work or inventions.

Reprint requests to: Siang Kok Sim, School of Mechanical and Production Engineering, Nanyang Technological University, Nanyang Avenue, Singapore 639798, Republic of Singapore. E-mail: msksim@ntu.edu.sg
3. Expressiveness related: the important factor is selfexpression. Whenever one expresses oneself in a unique or individualistic way, one is considered as being creative.

4. Psychoanalytic: creativity is defined in terms of the interaction between the id, ego, and the superego.

5. Process oriented: the emphasis is on the thinking process itself. That is, a certain type of process is said to characterize creativity.

Here, the perspective adopted is that creativity is to do with the thinking process itself. Perkins (1981) and Weisberg (1993) suggested that creativity is the outcome of ordinary thinking, only quantitatively different from everyday thinking, and does not necessarily require a qualitative leap or a creative spark. Weisberg summarized the issue: "Creative thinking is not an extraordinary form of thinking. Creative thinking becomes extraordinary because of what the thinker produces, not because the way in which the thinker produces it." Li (1996), in the development of the theory of conceptual intelligence, showed that conceptual thought and conceptual learning are the essence of human intelligence. Conceptual thought can lead to creative thinking, while conceptual learning, as opposed to behavioral learning, leads to knowledge acquisition. Hence, it can be argued that creative thinking and learning are related and linked to human intelligence and human cognition. Goldenburg and Mazur- 
sky (2002) posit the hypothesis that creative thinking is a process one may channel, diagnose, and reconstruct by use of analytical tools. Boden (1991) has reviewed computational approaches to creativity and presents examples of programs that can create art, find novel geometric proofs, discover scientific laws and principles, and design new products. These programs can generate outputs that in many cases seem surprisingly creative. Boden argues that such efforts have important implications for the understanding of human creativity, pointing out that computers, like people, can apply various rules or heuristics that can result in creative performance. Such examples provide impetus for this paper on creativity and learning in which one of aims is to understand the links between learning and creativity so that these can be computationally supported.

Complex systems are meaningfully characterized in terms of multiple description levels. The human cognitive system, the mind-brain entity, is arguably the most complex natural entity of which we are aware. DasGupta (1996) suggests that one of the ways in which we commonly attempt to grasp the complexity for the purpose of describing, explaining, or understanding mental processes is by recognizing that it can be abstracted at multiple level of descriptions. Each level is appropriate for a particular kind of inquiry into the cognitive processes. The broad levels of description for cognition recognized by cognitive scientists are the following:

1. knowledge level: cognition is described or explained in terms of goal, actions, knowledge, and intentionally rational behavior.

2. symbol level: cognition is described and explained in terms of symbols, memory, symbol-transforming operators, and interpretation of those operators.

3. biological level: cognition is described or explained in terms of biological structures.

Understanding cognition at the biological level proves to be intractable at the moment. Understanding cognition at the knowledge and symbol levels have been the basis of study of creativity by DasGupta (1994), learning by Michalski (1993) and Kocabas (1991), and learning in design by Sim (2000). The term knowledge level was coined by Newell (1982), who presented a systematic and detailed characterization of cognitive behavior at this level. Sim (2000) shows that learning and designing as cognitive processes can be abstracted at the knowledge level. Sim and Duffy (1998) presented that the cognitive activity of learning can be explained in terms of knowledge transformers. It is the thesis of this paper that these similar knowledge transformers are "cognitive mechanisms" by which the creative process can be characterized and explained. Hence, Section 2 presents the knowledge transformers as background knowledge, and suggests that some of the discoveries and inventions may be explained in terms of these transformers. Section 3 summarizes the accounts of some inventions and discoveries that led to the inventions with the view to explain these in terms of knowledge transformers. Section 4 reviews models and theories that attempt to explain creativity and the creative process. The review also identifies that the cognitive processes described therein are similar in their nature to the knowledge transformers. This identification provides the basis to suggest that learning and creativity are linked and Section 5 explores these links.

\section{KNOWLEDGE TRANSFORMERS}

Although human learning in general can be defined as a relatively permanent change in behavioral potential as the result of experience (Anderson, 1995), learning is viewed as a knowledge gaining process in a given context (i.e., design; Sim \& Duffy, 2004). Hence, a knowledge transformer is an operator that derives a piece of new knowledge from a given input or an existing piece of knowledge.

Sim and Duffy (1998) identify seven pair of main knowledge transformers that characterized the learning process in most Machine Learning in Design (MLinD) systems. These published MLinD systems have been developed to automate the learning of design knowledge (Sim \& Duffy, 1998). Although these knowledge transformations are implemented in terms of various machine learning methods, it is in the nature of the characteristics of each type of knowledge transformation that made their identification within the MLinD systems possible. Each machine learning method used may be either symbolic or subsymbolic in nature depending on the nature of the representation of the input/ output knowledge. The review of these MLinD and the identification of the knowledge transformers are given in some detail in Sim and Duffy (1998).

These knowledge transformers are similar to the seven pairs of knowledge generation transmutations of Michalski's (1993) Inferential Theory of Learning (ILT; which, in addition, has four knowledge manipulation transmutations). The objective of Michalski's ILT is to characterize the logical capabilities of learning methods and processes that he shows are founded on the logical inference of induction, deduction, and abduction. It is thus meant to be a rather general theory of learning that modifies the learner's knowledge by exploring the learner's experience. Arciszewski and Michalski (1994) applied the theory to show the knowledge change in the domain of design and called the transmutations, design knowledge transmutations. The knowledge transformers, like the knowledge generation transmutations, change the logical content of the input knowledge through the inference process of deduction, induction, or analogy. Hence, by substantiating that the knowledge transformers though derived from MLinD systems are a subset of the knowledge transmutations of ILT, Sim and Duffy (2004) argued that it is reasonable to suggest that these knowledge transformers provide the basis to model the types of learning in design. The seven pairs of knowledge transformers are 
Table 1. Knowledge transformers

\begin{tabular}{|c|c|}
\hline Knowledge Transformers & How Knowledge Is Transformed \\
\hline Abstraction/detailing & $\begin{array}{l}\text { Abstraction generates a less detailed description but with essential attributes } \\
\text { of the original target concept. Detailing is the opposite, in which more } \\
\text { details in terms of attributes are generated to the fuller description of the } \\
\text { target concept or between related or incompatible concepts. }\end{array}$ \\
\hline Association/disassociation & $\begin{array}{l}\text { Association determines a dependency between given entities or descriptions } \\
\text { based on some logical, causal, or statistical relationships. The opposite } \\
\text { is disassociation, in which there is a lack of dependency between the } \\
\text { entities. }\end{array}$ \\
\hline Derivations (reformulation)/randomization & $\begin{array}{l}\text { Derivations are transformations that determine knowledge from another } \\
\text { piece of knowledge (based on some dependency between them). In } \\
\text { reformulation, the dependency is that of logical equivalence; } \\
\text { reformulation transforms knowledge into a logically equivalent } \\
\text { knowledge. Randomization transforms one knowledge segment into } \\
\text { another by making random changes. }\end{array}$ \\
\hline Explanation/discovery & $\begin{array}{l}\text { Explanation derives additional knowledge based on prior domain } \\
\text { knowledge. That is, additional knowledge is compiled into an efficient } \\
\text { form (e.g., a proof tree) in order to explain (prove) the prior, domain } \\
\text { knowledge. Such additional knowledge could be part of the domain } \\
\text { knowledge. Without underlying, existing domain knowledge, discovery } \\
\text { derives new knowledge through hypothesis formation and hypothesis } \\
\text { testing. }\end{array}$ \\
\hline Group rationalization (or clustering)/decomposition & $\begin{array}{l}\text { Group rationalization involves the grouping of past designs according to } \\
\text { their similarities when considering particular perspective(s) or criteria. } \\
\text { Decomposition removes the groupings or creates subparts in elements } \\
\text { of a whole. }\end{array}$ \\
\hline Generalization/specialization & $\begin{array}{l}\text { Generalization generates a description that characterizes a concept based } \\
\text { on a conjunction of all the maximally general instances of that concept. } \\
\text { Generalization extends the reference set of that concept. Typically, the } \\
\text { underlying inference is inductive. Specialization increases the specificity } \\
\text { of the description that characterizes concepts based on maximally specific } \\
\text { instances of that concept. It reduces the reference set of that concept. }\end{array}$ \\
\hline Similarity comparison/dissimilarity comparison & $\begin{array}{l}\text { A similarity comparison derives new knowledge about a design on the } \\
\text { basis of similarity between the design and similar past design }(\mathrm{s}) \text {. It is } \\
\text { based on analogical inference. This process is also described as analogical } \\
\text { transfer or contextual shifting. The opposite is a dissimilarity comparison, } \\
\text { which derives new knowledge on the basis of a lack of similarity between } \\
\text { two or more past designs. }\end{array}$ \\
\hline
\end{tabular}

Adapted from Sim and Duffy (1998).

1. abstraction/detailing,

2. association/disassociation,

3. derivations (reformulation)/randomization,

4. explanation/discovery,

5. group rationalization (or clustering)/decomposition (ungroup),

6. generalization/specialization, and

7. similarity comparison/dissimilarity comparison.

They are further elaborated in Table 1.

\section{KNOWLEDGE TRANSFORMERS IN CREATIVITY}

As discussed in Section 2, because the knowledge transformers have their foundation in logical inference of induction, deduction, and abduction, the hypothesis proposed here is that these knowledge transformers can be linked to the thought process in creativity. By no means do these transformers alone embed creativity, but they serve to explain the complex process of creativity in terms of knowledge transformers that are more amendable to computational implementation of design support systems.

\subsection{Knowledge transformers in inventions and discovery}

The purpose of this section is to show some evidence that the discoveries in science and inventions in technology can be explained by knowledge transformers described in Section 2. This task is not without difficulties, as the inferences made in support of the transformers or the lack of these are dependent on the details and clarity by which the accounts of the discoveries or inventions were given by the authors themselves or as recorded by the historians. The second difficulty is that the terms or the phrases used to describe 
the inventions may have different meaning(s) to that of knowledge transformers given in Section 2. Recognizing these limitations, the purpose here is to glean through published books or journals that recorded these inventions and scientific discoveries that made these inventions possible. As this is a mammoth undertaking, what is elicited here cannot be claimed to be exhaustive.

Watson and Crick's discovery of the double-helical structure of DNA, perhaps the most important discovery of biology of the 20th century, occurred through analogical reasoning, hypotheses formation, and substantiation by experiments (Wallace \& Gruber, 1991; Strathern, 1997). The molecular modeling orientation that attracted Watson and Crick had as its most well-known chemist and practitioner Linus Pauling, who had scored a great triumph with a helical model that he proposed for the structure of protein $\alpha$-keratin, which forms many structures, including hair, horn, and fingernails. Pauling's working methods were of particular interest to Watson and Crick, because proteins are in many ways analogous to DNA: both are long-chain molecules, composed of building blocks, each joined to the next through chemical bonds. The building blocks of protein are the amino acids, while those of DNA are the nucleotides: a sugar, a phosphate, and one of the four nitrogen-rich bases (adenine, cytosine, guanine, and thymine). The double helix of DNA is constructed like a spiral staircase, with two sugar-phosphate chains connected by rungs made up of pair of bases.

The analogy in structure between protein and DNA leads to the possibility that Pauling's methods might be applicable to the analysis of DNA, and in the fall of 1951, soon after Watson arrived in Cambridge, he and Crick decided that they would try to build a helical model of DNA. Two specific problems arose in constructing the model: how many helical strands it should contain, and where the bases should be located. The available information did not make clear the quantities or locations of the parts of the molecule, although it was known that the molecule was wider than a single-stranded helix. In Watson and Crick's initial model, there were three strands rather than the correct two, and the bases projected outward from the backbones rather than being between them. Three strands were held together by bonds between magnesium ions. The initial insight that Watson and Crick had for the structure of the DNA can be explained by the cognitive process of analogical transfer. Analogical transfer depicts situations in which information from a previous situation is transferred to a new one that is analogous to the old; the new situation is similar in structure to the old one. The knowledge transformer that described the analogical transfer is similarity comparison.

Another example of similarity comparison is provided by Design Continuum, which used an old idea in a new way to develop an innovative medical product for cleansing wounds (Sutton, 2002). It was to be used in emergency rooms to clean wounds with a pulsating flow of saline solution. The new product, called a pulsed lavage, had to meet strict guidelines for cleanliness and safety. It had to be low cost and disposable. The Design Continuum engineers rec- ognized similarities between the pulsed lavage and a batterypowered squirt gun. On the surface, an emergency-room tool and the children's toy seem unrelated. However, once these engineers recognized the similarities between the two products, they were inspired to modify the inexpensive electric pump and battery of the squirt gun to meet the guidelines for the new medical product.

Table 2 summarizes other important inventions that have changed our lives over the past century and the knowledge transformers that are inferred from the published accounts of these inventions.

\section{MODELS AND THEORIES OF CREATIVITY}

The purpose of this section is to investigate whether there exist cognitive processes similar to knowledge transformers in the formulations of models and theories of creativity. This is based on the premise that proponents of these models or theories must characterize the essence of the cognitive process of creativity to explain the phenomenon of creativity. Only theories and models that explain the cognitive process of creativity have been selected for the investigation. Hence, the approach here is to infer/identify within the descriptions of the models and theories the presence of cognitive processes that are similar or synonymous with the knowledge transformers presented in Section 2.

\subsection{Bisociation}

Bisociation is the name given by Arthur Koestler (1964) in his book The Act of Creation to the notion that the essential ingredient in ideation is the effective combination of possible disparate or unconnected ideas: "elements drawn from domains which are far apart," in Poincare's words. ${ }^{1}$ Koestler associated creativity with unconscious thought, more precisely, with the unconscious combining of ideas. However, bisociation is not merely the combination of ideas but of ideas from different "planes," from "frames of references," or the term Koestler meant "any ability, habit or skill, any pattern of ordered behavior governed by code of fixed rules." Bisociation entails the linkage of (at least) two normally incompatible or unrelated matrices. For example, Maurice Wilkes' invention of microprogramming (Wilkes, 1986), signifies both a technique for designing the control unit of a computer as well as an architecture for control units. It entailed the combination of two entirely unrelated concepts, namely, the concept of programming on the one hand and a particular kind of circuit structure called a diode matrix on the other (DasGupta, 1994).

The knowledge transformers that can be inferred here are that of association/disassociation that Koestler (1964) referred to as bisociation in which unrelated or incompatible concepts are linked together.

\footnotetext{
${ }^{1}$ Koestler quoted from the lecture delivered by Poincare in 1908, but no reference is given in Koestler's book, The Act of Creation.
} 
Table 2. Inference of knowledge transformers through an account of selected inventions

\begin{tabular}{|c|c|c|}
\hline Invention & Summary of Inventions & Inferred Knowledge Transformer \\
\hline Microwave oven & $\begin{array}{l}\text { Shortly after World War II, Percy Spencer, an inventor, was touring one of } \\
\text { his laboratories at the Raytheon Company. Spencer stopped in front of a } \\
\text { magnetron, the power tube that drives a radar set. Suddenly he noticed } \\
\text { that a candy bar in his pocket had began to melt. Instead of wiping the } \\
\text { melted chocolate off his hands and shrugging off the incident, he asked } \\
\text { for popcorn. Holding a bag of unpopped kernels next to the magnetron, }\end{array}$ & $\begin{array}{l}\text { By associating the magnetron with cooking, } \\
\text { Spencer put a radar set disguised as a gigantic } \\
\text { microwave oven as an essential modern compact } \\
\text { household item, which evolved over the years to } \\
\text { its present size through solving problems of } \\
\text { overheating. }\end{array}$ \\
\hline
\end{tabular}

Telephone With the installation of the duplex telegraph system in 1872, Alexander Graham Bell's hypothesis was that a system that could transmit a dozen or more tones per wire was really in a tuning fork that he had experimented with as a youth. Bell studied the work of the German physicist Hermann von Helmholtz, who invented an electric tuning fork. By hooking up a tuning fork to an electromagnet and plucking the fork, Helmholtz found that an electric current vibrating at the same frequency as the fork could be sent through the wire.

By hooking up many tuning forks to a wire, diverse notes at a unique pitch were produced. To solve the problem of interference between signals produced by the forks, Bell developed a set of receiving reeds attached to the end of the wire. Each reed, turned to the exact frequency of each sending fork, would respond only to its own frequency. Hence, the number of signals that could be sent over the wire was limited only to the number of notes on the diatonic scale. This arrangement led to the invention of the harmonic telegraph by Bell (Flatow, 1992).

Xerox Photography involves mixing chemicals, a rather wet and messy process that takes hours to produce a print. Recognizing that photographic duplication was too impractical to be applied to the office, Chester Carlson developed an idea for a dry reproduction technique based on photoconductivity. Certain materials change their electrical properties when exposed to light. Simply charge up a photoconductive metal plate with electrostatic charges and project an image onto it. Where light from the image hits the plate, the charge totally disappears or is lessened, depending on the intensity of the light. Dust the plate with oppositely charged powder, so it sticks to the oppositely charged areas. The plate now has a record of the image in powder. By baking the image onto a sheet of paper, a dry reproduction is achieved. Carlson called this process electrophotography, and he received the patent in 1937. Haloid, which later became Xerox Corporation, bought a license to develop a dry copying machine based on Carlson's design (Flatow, 1992).

Velcro In 1948, Swiss engineer Georges de Mestral conceived the idea that evolved into Velcro after a walk in the woods. He came back home with burrs stuck to his socks and to his dog, and he decided to investigate why burrs stick so well to wool. Under the microscope, he saw how tiny hooks on the ends of the burrs caught in loops in the wool. De Mestral devised a method of reproducing the hook and loop arrangement in woven nylon. He called the product Velcro, a contraction of velours and crochet, the French word for "hook" (Flatow, 1992).

Walkman The earlier monophonic Pressman (a recording device) failed and was abandoned. Attempts to make it smaller also failed, because the recording system did not fit into its small size. By combining two seemingly unrelated projects at Sony Corporation, the incomplete tape recorder (Pressman) and unfinished lightweight portable headphones, Chairman Ibuka's suggestion led to the development of the Walkman. "What if you combined them?" asked Ibuka. At the very least, he said, the headphones would use battery power much more efficiently than stereo speakers. Reduce power requirements and you reduce battery consumption. But another idea began to form in his mind. If you added the headphones, wouldn't you dramatically increase the quality of what a listener hears? Could you leave out the recorder entirely and make a successful product that just plays music? (Nayak \& Ketteringham, 1986). Only after the integration of the Pressman with that of a light headphone was the Walkman concept defined.

Association of the tuning fork as the answer to the problem of transmitting a dozen or more tones per wire contributes to the invention of the harmonic telegraph.

Explanation of the cause and effect of the phenomenon of electric current vibrating at the same frequency as the fork

Explanation of cause and effect

By the keen observation of the burrs that stuck to his socks, through analogical inference, De Mestral developed a product called Velcro.

By combining two unrelated concepts (i.e., disassociation and association), Ibuka's suggestion led to the development of the Walkman. 


\subsection{The Darwinian model}

Donald Campbell proposed a Darwinian theory of the generation and growth of knowledge (Campbell, 1960). The application of Darwinian ideas to the resolution of particular problem has its foundation on the principle of natural selection. Keith Simonton (1988) proposed a Darwinian model of psychology of scientific discovery while Walter Vincenti (1990), the variation-selection theory. The evolutionary algorithms (e.g., genetic algorithms, genetic programming), based on the principle of natural selection, have become a well-established approach in solving problems involving multiobjective optimization.

The Darwinian perspective shed light on creativity, which can be summarized by its three mechanisms (Campbell, 1960). First, the production of genuinely new knowledge or ideas that are original demands the generation of variations that are blind in the sense that the consequences of these variations go beyond what can be foreseen or anticipated. Second, the variations are then subjected to a selection process that prunes out all variations that do not demonstrate a fit with the problem at hand. In the context of creative designs, this implies that one or more criteria are used to judge or rank alternative solutions, resulting in the rejection of those that fail to meet the criteria. Third, there must be a mechanism for the retention and propagation of the selected variation. This is because, without retention, a selected or successful variation can hardly make a permanent contribution to the acquisition and growth of knowledge. There is no knowledge transformer identified for the third mechanism, because it is more akin to that of storage (i.e., memory) of the selected variation.

The knowledge transformer inferred based on the first mechanism is that of randomization, which ensures generation of variations that are blind. The knowledge transformer inferred in the second mechanism is that of similarity comparison/dissimilarity comparison to prune variations that do not fit the problem under investigation.

\subsection{Geneplore model}

The Geneplore model consists of two distinct processing components: a generative phase, followed by an exploratory phase (Finke et al., 1995). In the initial, generative phase, one constructs mental representations called preinventive structures, having various properties that promote creative discovery. These properties are then exploited during an exploratory phase in which one seeks to interpret the preinventive structures in meaningful ways. These preinventive structures can be thought of as internal precursors to the final, externalized creative products, and would be generated, regenerated, and modified throughout the course of creative exploration.

\subsubsection{Generative processes}

The most basic types of generative processes consist of the retrieval of existing structures from memory and the for- mation of associations among these structures. A richer variety of preinventive structures result from the mental synthesis of component parts and by the mental transformation of the resulting forms. Parts can be mentally rearranged and reassembled, and forms can be rotated or altered in shape to make interesting and potentially useful structures. Single concepts can be combined to form more complex concepts, with the meanings of one or both of the initial concepts being altered as a result. Another type of generative process is analogical transfer, in which a relationship or set of relationships in one context is transferred to another, resulting in preinventive structures that are analogous to those that are already familiar. In the context of knowledge transformers, this is analogical transfer is synonymous with similarity comparison. Categorical reduction, another important generative process, means mentally reducing objects or elements to more primitive categorical descriptions.

\subsubsection{Exploratory processes}

The first example of exploratory processes is attribute finding, the systematic search for emergent features in the preinventive structures. Attribute finding could also be used to explore emergent features resulting from the creation of conceptual combinations and metaphors. Conceptual interpretation refers quite broadly to the process of taking a preinventive structure and finding an abstract, metaphorical, or theoretical interpretation of it. Functional inference refers to the process of exploring the potential uses or functions of a preinventive structure.

Another exploratory process is contextual shifting, in which one considers a preinventive structure in new or different context as a way of gaining insights about other possible uses or meanings of the structure. Preinventive structures can also be explored in the spirit of hypothesis testing, where one seeks to interpret the structures as representing possible solutions to a problem.

Yet, another exploratory process is searching for limitations. For example, when people generate exemplars for novel categories, they often discover that their initial creations are limited in important respects. They might then explore those limitations, leading to the creation of more appropriate exemplars.

\subsubsection{Related knowledge transformers}

In the generative phase, the knowledge transformers identified are association, analogical transfer, and abstraction, while in the exploratory phase they are abstraction, discovery through hypothesis formation (i.e., attribute finding, finding abstract, metaphorical, or theoretical interpretation of concepts, functional interpretation), and hypothesis testing.

\subsection{Li's theory of conceptual intelligence}

Li (1996) developed a theory of intelligence based on thinking and learning. He defines intelligence as follows: "Intelligence is the mental capacity for higher-order conceptual 


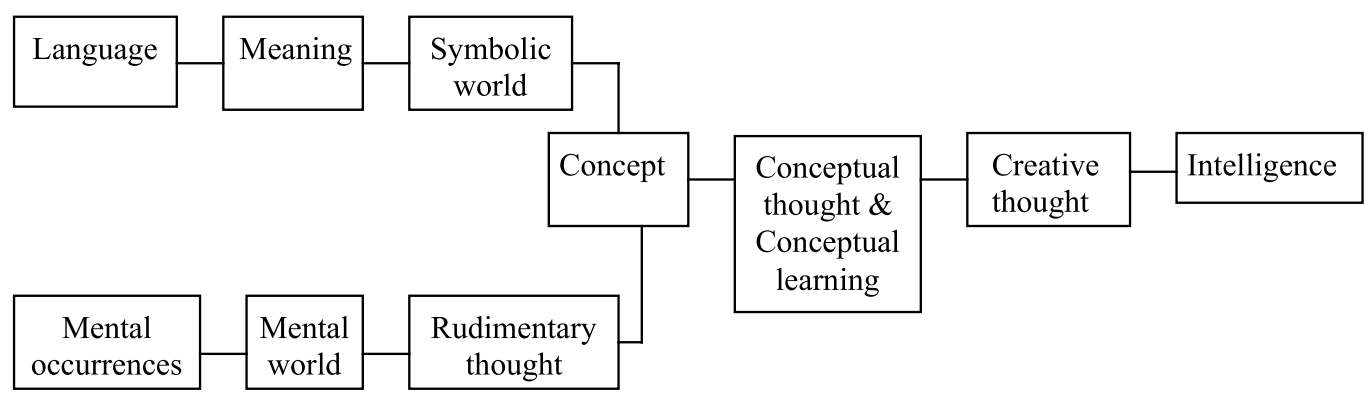

Fig. 1. The links between language and intelligence (adapted from Li, 1996).

activities of thinking and the acquisition of knowledge." Based on the assumption that intelligence has a strong link with symbolic systems, his conjecture is that intelligence is the unintended consequence of using symbol systems that facilitate thinking and learning. Recognizing that human beings use various symbolic vehicles (e.g., language, music, gesture, mathematics, and pictures; Gardner, 1983) in expressing and communicating meaning, Li suggests that it is language that has led to the creation of concepts that further enhance thinking and learning. The human language has led to the creation of a symbolic world, while the mental occurrences have brought about a mental world. The interactions of both worlds have transformed rudimentary thought into concepts. Once concepts are formed, thinking and higher order conceptual activities come into being, hence the emergence of intelligence (see Fig. 1).

Figure 1 suggests that thinking can be abstracted at three levels: rudimentary thought, conceptual thought, and creative thought ( $\mathrm{Li}, 1996)$. Li distinguishes between rudimentary and conceptual thought by suggesting that the former is carried out without the medium of language while the latter is. Rudimentary thought is very much dependent on the visual presence of physical objects, and hence limited to operation on physical objects and actions. Conceptual thought is made possible by the creation of concepts that are the interaction between the symbolic world and the mental world. Through the medium of language, higher order concepts such as relations between objects and qualities extracted from generalization of objects are possible through conceptual thinking.

Li (1996) considers conceptual thinking and conceptual learning as the two major unique characteristics of human intelligence. Although Li has developed a theory of conceptual intelligence, what is unique about the theory is the link between conceptual thought and conceptual learning to creativity. In fact, Li suggests that learning precedes creativity in that the discovery of new knowledge (i.e., the creative process) cannot possibly exist out of the blue; it can always be traced back to its roots in existing knowledge. He posits that in a problem-solving situation, a person is making use of a number of existing conventional frameworks of knowledge to bear on the problem. Li suggests that a transformation may take place leading to the creation of new knowledge, but he did not suggest how this transformation takes place.

In Li's (1996) example of a creative work by Jaynes in developing a theory to explain consciousness, he alluded to the fact that Jaynes combined all the subbranches of knowledge (i.e., philosophy, psychology, language, and history) that were configured and reorganized to the point of proposing a unifying theme in a meaningful way. He suggested that it is the important insight of the connection/similarity between the description of hallucinating schizophrenics and the mind of the ancient people as obedient to the voices of God that led him to gather evidence from different knowledge domains for the development of his theory. Although Li did not suggest the nature of the transformation, here in this study we infer that the knowledge transformer inferred is that of a similarity comparison.

\subsection{Summary}

Table 3 shows a summary of the identification of the knowledge transformers in the various models and theory of creativity.

\section{DISCUSSION}

The purpose of this paper is to show that learning and creativity are linked through knowledge transformers that Sim and Duffy (1998) identified as learning operators that derive new knowledge through learning in design.

To show the link, first, it was shown in Section 3 that some scientific discoveries and inventions can be explained in terms of the knowledge transformers. Although the review was not exhaustive, Table 2 shows that in the description of the scientific discovery or inventions certain knowledge transformers have been inferred or identified.

Second, it was shown in Section 4 that some of the knowledge transformers are identified in the models and a theory of cognitive process of creativity. Mumford et al. (1991) and Brophy (1998) suggest an interplay between divergent (ideation) and convergent (evaluative) modes of thought in creativity. It is apparent from the descriptions of these models that the cognitive process of creativity entails divergent and convergent phases. Hence, it is conceivable that mod- 
Table 3. Identification of knowledge transformers in models or theories of creativity

\begin{tabular}{lcccc}
\hline \multicolumn{1}{c}{ Knowledge Transformers } & $\begin{array}{c}\text { Bisociation Model } \\
\text { (1964) }\end{array}$ & $\begin{array}{c}\text { Darwinian Model } \\
\text { (1960) }\end{array}$ & $\begin{array}{c}\text { Geneplore Model } \\
\text { (1995) }\end{array}$ & $\begin{array}{c}\text { Li Theory } \\
(1996)\end{array}$ \\
\hline Abstraction/detailing & & & \\
Association/disassociation & & & \\
Derivations (e.g., reformulation)/randomization & & & \\
Explanation/discovery \\
Group rationalization (or clustering)/decomposition \\
Generalization/specialization \\
Similarity comparison/dissimilarity comparison
\end{tabular}

els or theories that describes cognitive process of creativity entail divergent and convergent phases.

Koestler's model of creativity (1964) describes the divergent phase in which creative ideas can emerge from an effective combination of possible disparate or unconnected ideas or independent matrices (i.e., bisociation). Although the concept by bisociation is a combination of independent matrices that give rise to novelty, the concept of association is achieved by combination within the confines of a given matrix. Thus, bisociation is akin to association in its process but not in its content. Hence, the knowledge transformer identified here is that of association/disassociation.

In the Darwinian model, the first mechanism provides a diverging insight generating variations beyond what is foreseen or anticipated. The second mechanism provides a converging insight to the problem by pruning or ranking solutions to a set of criteria. The knowledge transformers identified are randomization and similarity comparison, respectively.

In Geneplore, in the generative or divergent phase where the aim is to generate preinventive structures to promote discovery, the knowledge transformers identified are association, analogical transfer, and abstraction. In the exploratory or converging phase where the aim is interpret these preinventive structures in meaningful ways, the knowledge transformers identified are discovery through hypothesis formation through attribute finding, finding abstract, metaphorical, or theoretical interpretation of concepts, functional interpretation, and then hypothesis testing through contextual shifting.

From the evaluation of the three models, it can be suggested that the knowledge transformers identified in the divergent phase are association/disassociation, randomization, analogical transfer, and abstraction. For the convergent phase, the knowledge transformers identified are similarity comparison and discovery through hypothesis formation and testing. These inferences are not conclusive due to the limited number of models reviewed.

Another interesting inference provided in Table 3 is that two pairs of knowledge transformers may not be featured in the models and theory. They are group rationalization (clustering)/decomposition and generalization/speciali- zation. These knowledge transformers are more predominant in processes related to routine or variant designs where base concepts/solutions are known in advance for these processes to operate.

Li (1996) suggests that learning precedes creativity in the discovery of new knowledge, and alluded to the concept of knowledge transformation that may lead to the creation of new knowledge. However, he did not suggest how this transformation takes place. Li's theory of conceptual intelligence has been included in the investigation because it shows a link between creativity and learning. As explained by $\mathrm{Li}$, creativity can be seen as the human enterprise of extending from the known to the unknown, of venturing from existing knowledge and domains of human endeavor to new knowledge and endeavor. Although learning is the acquisition of existing knowledge and skills, creativity is the search for new knowledge and new skills. Although the same cognitive apparatus (human mind) is used to acquire knowledge as well as creating knowledge, the task demand is quite different. In acquiring existing knowledge, the subject matter is well structured and well organized. The same cannot be said when we venture beyond the existing realm of knowledge in which there is no clear definition of the problem let alone the solution. Creativity is about discovery and reasoning of what is observed, and sometimes discovery leading to invention is made in a accidental manner (e.g., the invention of Velcro). To Fisher and Nakakoji (1997), creativity in design plays a critical role in cultivating learning in design activities. Although creativity and learning are distinct thought processes, from the limited evidence given, learning proceeds from known knowledge to new knowledge while creativity proceeds from the unknown to discover new knowledge.

From the discussion above, there is some evidence to suggest that the knowledge transformers featured in the cognitive activity of learning by Sim and Duffy (1998) have been identified in the models and a theory of creativity reviewed in Section 4. Because of the limited number of models and theory discussed in Section 4, it is difficult to conclude that these knowledge transformers can be identified in other models or theories as well. Conversely, it is difficult to conclude that perhaps there are models or theo- 
ries that do not feature any of these transformers. Nevertheless, with the evidence presented in Section 3 and 4, it appears that the thought process as explained through the knowledge transformers is the same regardless of whether one is engaged in the learning or creative process. Hence, it may be reasonable to suggest that creativity and learning are linked through the knowledge transformers.

\section{CONCLUSION}

The purpose of this paper is to investigate whether knowledge transformers that are featured in the learning process are also present in the creative process. The investigation shows that there is some evidence to show that the creative process can be explained through knowledge transformers. Thus, we suggested that one of links between learning and creativity is through the knowledge transformers. Further work is required to substantiate this link so that suitable computational support can be built to facilitate both learning and creativity in design.

\section{ACKNOWLEDGMENTS}

The first author expresses his gratitude to the Dean of the School of Mechanical \& Production Engineering, Nanyang Technological University, for the opportunity to spend a 6-month sabbatical at the Engineering Design Centre (EDC), University of Cambridge, and to Dr. P. John Clarkson, the Director of EDC, for making the stay at EDC a fruitful and enjoyable one, during which the initial draft of this paper was written.

\section{REFERENCES}

Anderson, J.R. (1993). Problem solving and learning. American Psychologist $48,35-44$.

Arciszewski, T., \& Michalski, R.S. (1994). Inferential Design Theory: A conceptual outline. In Artificial Intelligence in Design '94, pp. 295308. Boston: Kluwer Academic.

Boden, M. (1991). The Creative Mind: Myths and Mechanisms. New York: Basic Books.

Brody, D.R. (1998). Understanding, measuring and enhancing individual creative problem-solving efforts. Creativity Research Journal 11, 123-150.

Campbell, D.T. (1960). Blind variation and selective retention in creative thoughts as in other knowledge processes. Psychological Review 67, 380-400.

DasGupta, S. (1994). Creativity in Invention and Design-Computational and Cognitive Explorations of Technological Originality. New York: Cambridge University Press.

DasGupta, S. (1996). Technology and Creativity. New York: Oxford University Press.

Finke, R.A., Ward, T.B., \& Smith, S.M. (1995). The Creative Cognition Approach. Cambridge, MA: MIT Press.

Fisher, G., \& Nakakoji, K. (1997). Computational environments supporting creativity in the context of lifelong learning and design. KnowledgeBased Systems 10, 21-28.

Flatow, I. (1992). They All Laughed . . From Light Bulbs to Lasers: The Fascinating Stories Behind the Great Inventions That Have Changed Their Lives. New York: Harper Collins.

Gardner, H. (1983). Frame of Minds: The Theory of Multiple Intelligence. New York: Basic Books.

Goldenburg, J., \& Mazursky, D. (2002). Creativity in Product Innovation. New York: Cambridge University Press.
Kocabas, S. (1991). A review of learning. The Knowledge Engineering Review 6(3), 195-222.

Koestler, A. (1964). The Act of Creation. London: Hutchinson \& Co.

Li, R. (1996). A Theory of Conceptual Intelligence. New York: Praeger.

Nayak, P.R., \& Ketteringham, J.M. (1986). Breakthroughs! New York: Rawson Associates.

Newell, A. (1982). The knowledge level. Artificial Intelligence 18, 87-127.

Michalski, R.S. (1993). Inferential theory of learning as a conceptual basis for multi-strategy learning. Machine Learning 11, 3-43.

Mumford, M.D., Mobley, M.I., Uhlman, C.E., Reiter-Palmon, R., \& Doares, L.M. (1991). Process analytic models of creative capacities. Creativity Research Journal 4, 91-122.

Perkins, D.N. (1981). The Mind's Best Work. Cambridge, MA: Harvard University Press.

Sim, S.K. (2000). Modelling learning in design. PhD Thesis. Glasgow, UK: University of Strathclyde.

Sim, S.K., \& Duffy, A.H.B. (1998). A foundation for machine learning in design. Artificial Intelligence for Engineering Design, Analysis and Manufacturing 12, 193-209.

Sim, S.K., \& Duffy, A.H.B. (2004). Evolving a model of learning in design. Research in Engineering Design 15, 40-61.

Simonton, D.K. (1988). Scientific Genius: A Psychology of Science. Cambridge: Cambridge University Press.

Strathern, P. (1997). Crick, Watson \& DNA. London: Arrow.

Sutton, R.I. (2002). Weird Ideas That Work, p. 26. New York: Free Press.

Taylor, C.W. (1988). Various approaches to and definitions of creativity. In The Nature of Creativity (Sternberg, R.J., Ed.). New York: Cambridge University Press.

Vincenti, W.G. (1990). What Engineers Know and How They Know It. Baltimore, MD: Johns Hopkins University Press.

Wallace, D.B., \& Gruber, H.E. (1992). Creative People at Work: Twelve Cognitive Case Studies. New York: Oxford University Press.

Weisberg, R.W. (1993). Creativity: Beyond the Myth of Genius. San Francisco, CA: Freeman.

Wilkes, M.V. (1986). The genesis of microprogramming. Annals of the History of Computing 8(2), 116-118.

Siang-Kok Sim worked as a Naval Architect in a Singaporebased British company involved in the design and construction of military vessels before joining Nanyang Technological University (NTU), Singapore. He holds a BS (First Honors) from the University of Newcastle upon Tyne, an MS (intelligent knowledge-based systems) from the University of Essex, and a PhD (in learning in design) from the University of Strathclyde, Glasgow. Dr. Sim is presently an Associate Professor at NTU, where his main research interests are in the areas of artificial intelligence in design and manufacturing, design ontology, MLinD, robot learning, and neural networks.

Alex H.B. Duffy completed a shipwright draughtsman apprenticeship and a further 2 years in shipbuilding before he obtained a degree in naval architecture and a $\mathrm{PhD}$ in knowledge-based computer support for conceptual engineering design. He is presently Director of the Computer Aided Design Centre and the Director of Research in the Department of Design Manufacture and Engineering Management at the University of Strathclyde. Dr. Duffy's main research interests have been the application of knowledgebased techniques in early-stage design, product and domain knowledge modeling, learning in design and reuse, performance measurement and design productivity, process optimization, vague geometric modeling and sketching support, and design coordination. 